\title{
Thermal properties of nanocrystalline cellulose and cellulose nanowhisker
}

\author{
Lau Kia Kian, Mohammad Jawaid
}

\begin{abstract}
Nanocellulose is a renewable and sustainable biomass product which could be derived from natural fibers for various applications. At present study, nanocrystalline cellulose (NCC) and cellulose nanowhisker (CNW) are extracted from roselle fiber through different combination of acid hydrolysis and ultrasonication processes. The thermal properties of both types nanocellulose are compared in order to study their different decomposition behavior towards high temperature. From thermogravimetric analysis (TGA) results, NCCs had significantly lower decomposition temperature than that of CNWs, particularly those extracted under mild condition of the combined processes. However, under harsh condition of the combined processes, the derivative thermogravimetric (DTG) curve revealed decrement in peak decomposition temperatures for both NCC and CNW products. This was in line with the sequential occurrence of endothermic and exothermic behaviors as presented in differential scanning calorimetry (DSC) curve analysis. Hence, as evaluated from the overall comparative results, CNW could be utilized as a promising reinforcing agent for composite materials fabrication.
\end{abstract}

Keywords: nanocellulose, cellulose nanowhisker, nanocrystalline cellulose, thermal property

\section{INTRODUCTION}

Natural fiber is a sustainable and renewable biomass that can be widely obtained from agro-waste products. Recently, a derivative of natural fiber, nanocellulose has become a potential filling agent for strengthening polymer matrix through its high specific surface area for interfacial adhesion [1]. The growing of environmental awareness inspired those scientists and researchers to deeply explore the processing techniques required for producing nanocellulose with optimal properties from natural fibers. Also, due to the compelling behavior of nanocellulose like non-toxicity, biocompatibility, hydrophilic reactivity as well as self-assembling in liquid state, it has been extensively utilized as thickener, stabilizer and emulsifier in pharmaceutical, cosmetics and food applications [2]. As for heavy duty fields, nanocellulose was mostly employed as reinforcing agents in aerospace, automobile and some biomedical industry for manufacturing high performance materials by which the stable thermal

Revised Manuscript Received on September 12, 2019.

* Correspondence Author

Lau Kia Kian, Department of Biocomposite Technology, Institute of Tropical Forestry and Forest Products, Universiti Putra Malaysia, UPM Serdang, Selangor, Malaysia. Email: laukiakian@gmail.com

Mohammad Jawaid*, Department of Biocomposite Technology, Institute of Tropical Forestry and Forest Products, Universiti Putra Malaysia, UPM Serdang, Selangor, Malaysia. Email: jawaid_md@yahoo.co.in property of nanocellulose particles are highly requested [3]. Since that, the applicability of nanocellulose for high temperature fabrication process is crucial for most industrial fields [4].

At present work, it focuses on investigating the thermal property of nanocellulose. The most commonly known nanocellulose particles are nanocrystalline cellulose (NCC) and cellulose nanowhisker $(\mathrm{CNW})$, which inherent the biodegradable nature of cellulose attributed from retained intact of crystalline segments during acid hydrolysis (Santos et al., 2017). Both types of nanocellulose were produced from roselle fiber by employing different combination of acid hydrolysis and sonication techniques. Thermal stability examination was carried out on the produced nanocellulose using thermogravimetric analysis (TGA), derivative thermogravimetric (DTG), and differential scanning calorimetry (DSC).

\section{METHODOLOGY}

Roselle fiber was used as starting material for NCC and $\mathrm{CNW}$ isolations. Bleaching treatment was conducted for $1 \mathrm{~h}$ at $70-80^{\circ} \mathrm{C}$ with sodium hypochlorite $(\mathrm{NaCIO})$ solution that acidified with acetic acid to $\mathrm{pH} 4$. Then, the bleached fiber was treated with an $8.0 \%$ (w/v) sodium hydroxide $(\mathrm{NaOH})$ solution for $30 \mathrm{~min}$ at room temperature. The alkali treated bleached pulp was filtrated, washed and dried in an oven at $60^{\circ} \mathrm{C}$ for $24 \mathrm{~h}$. After that, the pulp thus obtained was undergone the combined acid hydrolysis and sonication treatments as shown in Table I.

Table-I: Combined treatments of hydrolysis and sonication for nanocellulose and its denotations.

\begin{tabular}{llll}
\hline Nanocellulose & Denotations & $\begin{array}{l}\text { Hydrolysis } \\
\text { time (min) }\end{array}$ & $\begin{array}{l}\text { Sonication } \\
\text { amplitude (\%) }\end{array}$ \\
\hline $\begin{array}{l}\text { Nanocrystalline } \\
\text { cellulose }\end{array}$ & NCC-I & 45 & 40 \\
$\begin{array}{l}\text { Cellulose } \\
\text { nanowhisker }\end{array}$ & CNW-I & 45 & 40 \\
& CNW-II & 45 & 30 \\
\hline
\end{tabular}


Afterwards, the suspension was left for 15 min to settle down the undesired large cellulose component. Finally, the supernatant nanocellulose suspension was collected and underwent freeze drying for thermal analyses.

\section{RESULTS AND DISCUSSION}

The TGA curves of nanocellulose samples were presented in Fig. 1. Both NCCs and CNWs revealed initial weight loss in temperature range of $70-130^{\circ} \mathrm{C}$, which were attributed to the evaporated water content. Beyond $200^{\circ} \mathrm{C}$ temperature, NCCs revealed lower onset decomposition temperatures when comparing to CNWs. It was possibly due to the different cellulose arrangement that affected the resistance behavior to heat degradation [5]. Meanwhile, the NCC-I $\left(287.7^{\circ} \mathrm{C}\right)$ and $\mathrm{CNW}-\mathrm{I}\left(372.6^{\circ} \mathrm{C}\right)$ samples had higher onset decomposition temperature than the corresponding NCC-II $\left(243.1^{\circ} \mathrm{C}\right)$ and $\mathrm{CNW}$-II $\left(361.5^{\circ} \mathrm{C}\right)$ samples (Table II). In accordance with reported studies, it was related to the generation of thermally unstable cellulose structure when subjecting to harsh condition [6]. Samples of CNW exhibited greater onset decomposition temperature when comparing to NCC samples. Also, the significant difference of onset decomposition temperatures was more obvious occurring in between CNW-I and CNW-II as compared to between NCC-I and NCC-II. It showcased that the reduction of ultrasonic amplitude below $40 \%$ could produce higher thermal stability of nanocellulose when comparing to the decrement of hydrolysis times. Furthermore, CNWs revealed more residue formation and less total weight loss as compared to NCCs. This indicated that the CNWs crystalline structure in relating to its rigidity were stronger than that of NCCs [7]. Moreover, CNWs exhibited well-resolved DTG curves when in comparison with NCCs as shown in Fig. 2. The peak decomposition temperatures for NCCs were in the range of 298.9-303. $4^{\circ} \mathrm{C}$, while CNWs were in the range of $360.3-362.2^{\circ} \mathrm{C}$ (Table II). This implied the different combined acid hydrolysis and sonication techniques could largely influence the nanocellulose thermal degradation. Also, those nanocellulose samples showed only one-stage decomposition, signaling the high purity of cellulose component [8].

Besides that, the heat degradation of cellulose component could be analyzed through the DSC curves as illustrated in Fig. 3. The broad endothermic peaks located at around $90^{\circ} \mathrm{C}$ for all nanocellulose showing the phenomenon of heat absorption for water evaporation process [9].

At $200^{\circ} \mathrm{C}$ temperature, a small second endothermic peak prevailed and it representing the thermal degradation had begun in the cellulose. The onset degradation temperature was in the range of $277.32-289.57^{\circ} \mathrm{C}$ for NCC samples, while it was in the range of $290.23-294.64^{\circ} \mathrm{C}$ for $\mathrm{CNW}$ samples (Table III). Those obtained DSC values were nearly closed to the data from TGA and this verified that the DSC analysis was in line with TGA analysis.

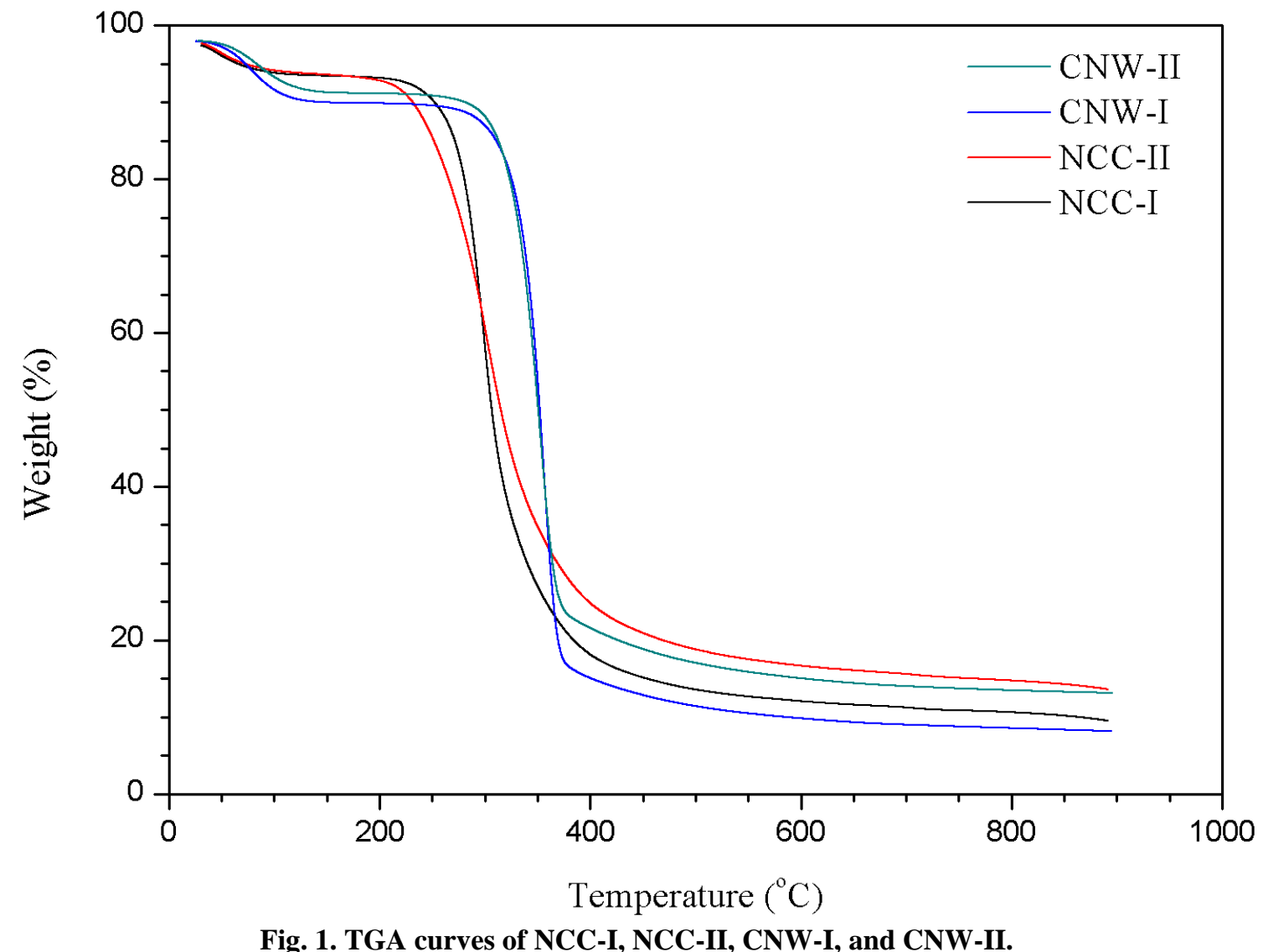

Fig. 1. TGA curves of NCC-I, NCC-II, CNW-I, and CNW-II. 


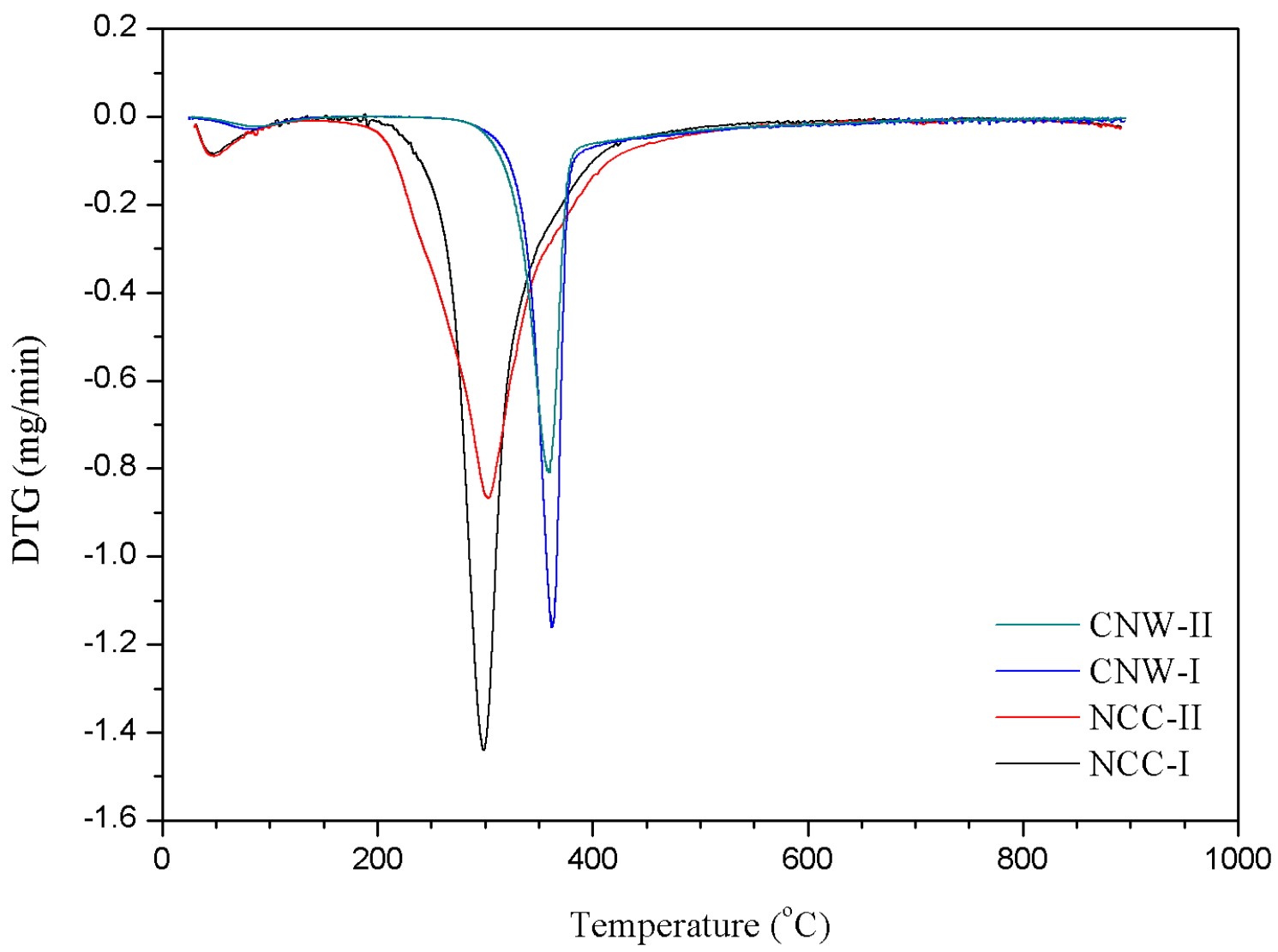

Fig. 2. DTG curves of NCC-I, NCC-II, CNW-I, and CNW-II.

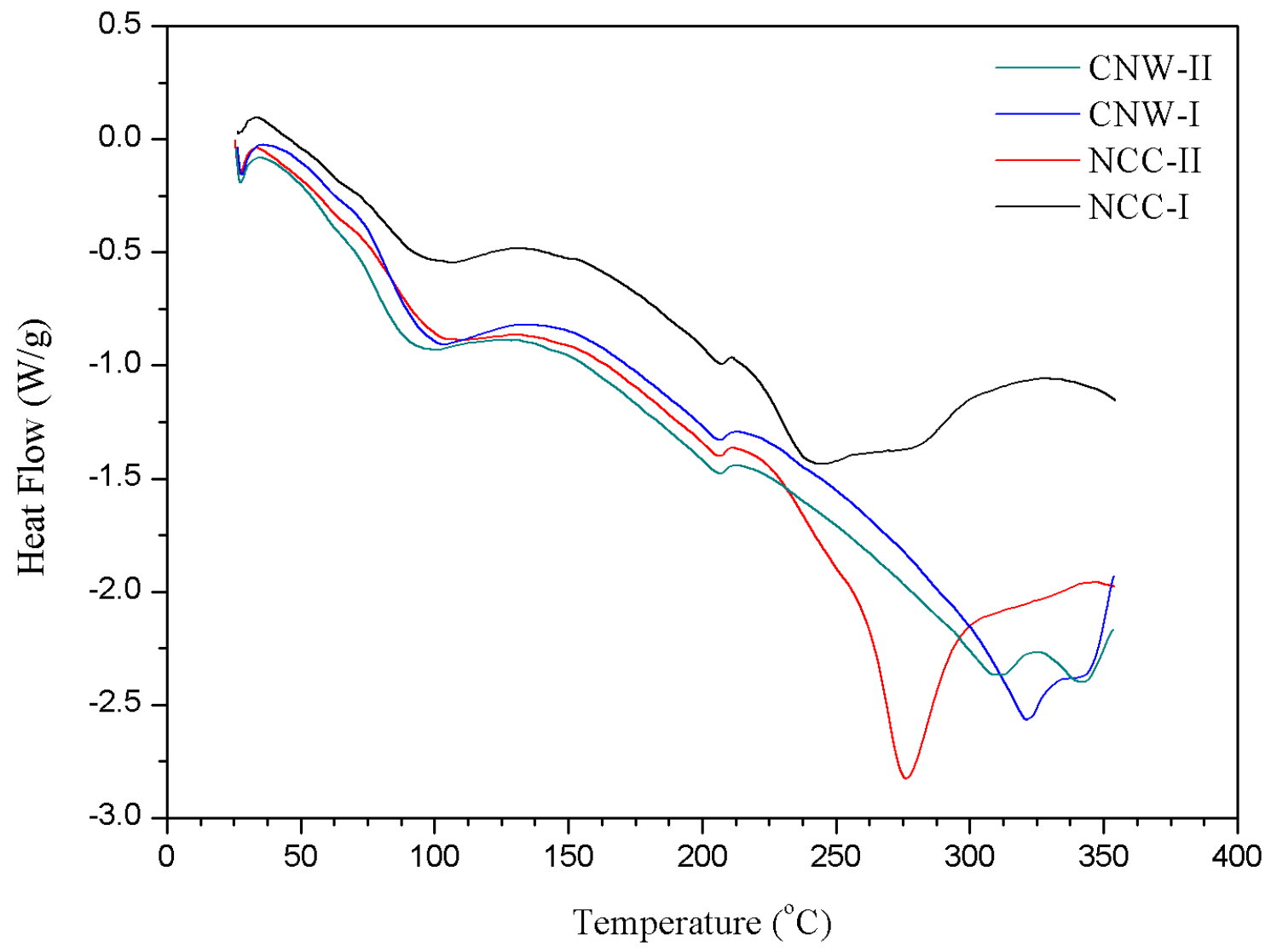

Fig. 3. DSC curves of NCC-I, NCC-II, CNW-I, and CNW-II. 
Table-II: TGA data of nanocellulose.

\begin{tabular}{|c|c|c|c|c|}
\hline \multirow[t]{2}{*}{ Samples } & \multicolumn{4}{|c|}{ TGA analysis } \\
\hline & $\mathrm{T}_{\text {onset }}\left({ }^{\circ} \mathrm{C}\right)^{\mathrm{a}}$ & $\mathrm{T}_{\text {peak }}\left({ }^{\circ} \mathrm{C}\right)^{\mathrm{b}}$ & $\mathrm{W}_{\text {loss }}(\%)^{\mathrm{c}}$ & $\mathrm{W}_{\text {residue }}(\%)^{\mathrm{d}}$ \\
\hline NCC-I & 287.7 & 303.4 & 89.14 & 9.81 \\
\hline NCC-II & 243.1 & 298.9 & 89.02 & 9.94 \\
\hline CNW-I & 372.6 & 362.2 & 87.63 & 11.25 \\
\hline CNW-II & 365.5 & 360.3 & 86.98 & 12.06 \\
\hline
\end{tabular}

${ }^{\mathrm{a}}$ onset decomposition temperature; ${ }^{\mathrm{b}}$ DTG maximum weight loss peak temperature; ${ }^{\mathrm{c}}$ weight loss; ${ }^{\mathrm{d}}$ char residual weight.

Table-III: DSC data of nanocellulose.

\begin{tabular}{llll}
\hline Samples & \multicolumn{2}{l}{ DSC analysis } & \\
\cline { 2 - 4 } & $\mathrm{T}_{\text {onset }}\left({ }^{\circ} \mathrm{C}\right)^{\mathrm{a}}$ & $\mathrm{T}_{\text {peak }}\left({ }^{\circ} \mathrm{C}\right)^{\mathrm{b}}$ & $\Delta \mathrm{H}(\mathrm{J} / \mathrm{g})^{\mathrm{c}}$ \\
\hline NCC-I & 289.57 & 325.62 & 79.73 \\
NCC-II & 277.32 & 323.76 & 92.95 \\
CNW-I & 294.64 & 343.39 & 74.22 \\
CNW-II & 290.23 & 341.61 & 76.51
\end{tabular}

${ }^{\mathrm{a}}$ onset degradation temperature; ${ }^{\mathrm{b}}$ exothermic peak temperature; ${ }^{\mathrm{c}}$ heat of degradation.

In later stage, the appearance of a series of exotherms and endotherms had given the signs that the occurrence of depolymerization, decarboxylation, and volitalization in cellulose components [10]. Noticeably, in this region, the NCC-II sample revealed a remarkable large broad endothermic peak when compared to NCC-I. It was probably owing to the presence of sulfated surface component as a result of prolonged acid hydrolysis treatment had somehow distorted the degradation behavior of NCC-II. From Table III, the heat enthalpy required for degrading cellulose component had increased tremendously from NCC-I $(79.73 \mathrm{~J} / \mathrm{g})$ to NCC-II $(92.95 \mathrm{~J} / \mathrm{g})$, while the NCC-I had closely enthalpy as with CNW-I $(74.22 \mathrm{~J} / \mathrm{g})$ and $\mathrm{CNW}-\mathrm{II}(76.51 \mathrm{~J} / \mathrm{g})$. This showed that the NCC-II had unusual thermal behavior when comparing to NCC-I. Between CNW-I and CNW-II, both samples exhibited relatively similar degradation patterns, indicating the changes of ultrasonic amplitudes only slightly affected the thermal behavior of nanocellulose [11]. However, the CNWs samples presented better cellulose degradation patterns than the NCCs samples. This could prove that the thermal decomposition was more stable for CNWs samples. Additionally, the peak degradation temperatures analyzed by DSC were within the range of 298.9-362. $2^{\circ} \mathrm{C}$, indicating the results were in agreement with the data as analyzed by DTG.

\section{CONCLUSION}

The major findings of present study revealed that the different combined acid hydrolysis and sonication techniques could isolate both NCC and CNW products with varying thermal behavior. In comparative analysis, the TGA and DTG curves presented the onset decomposition temperatures and peak decomposition temperatures were found greater for CNWs when comparing to NCCs. This endowed the CNWs with good heat tolerance capability that suitable for utilization in high temperature composite fabrication process. Moreover, the DSC analysis had revealed the heat degradation were relatively stable for CNWs, suggesting it was applicable for manufacturing high performance products in biomedical, engineering and electronic industries.

\section{REFERENCES}

1. L. Jasmani, \& S. Adnan. (2017). Preparation and characterization of nanocrystalline cellulose from Acacia mangium and its reinforcement potential. Carbohydrate Polymers, 161, 166-171.

2. X. Y. Qiu, \& S. W. Hu. (2013). "Smart" materials based on cellulose: a review of the preparations properties, and applications. Materials, 6, 738-781.

3. R. Prathapan, R. Thapa, G. Garnier, \& R. F. Tabor. (2016). Modulating the zeta potential of cellulose nanocrystals using salts and surfactants. Colloids and Surfaces A: Physicochemical and Engineering Aspects, 509, 11-18. 
4. E. Espino, M. Cakir, S. Domenek, A. D. R. Gutierrez, N. Belgacem, \& J. Bras. (2014). Isolation and characterization of cellulose nanocrystals from industrial by-products of Agave tequilana and barley. Industrial Crops and Products, 62, 552-559.

5. T. Taflick, L. A. Schwendler, S. M. L. Rosa, C. I. D. Bica, \& S.M.B. Nachtigall. (2017). Cellulose nanocrystals from acacia bark-influence of solvent extraction. International Journal of Biological Macromolecules, 101, 553-561.

6. M. Mariano, R. Cercena, \& V. Soldi. (2016). Thermal characterization of cellulose nanocrystals isolated from sisal fibers using acid hydrolysis. Industrial Crops and Products, 94, 454-462.

7. J. Lamaming, R. Hashim, O. Sulaiman, C. P. Leh, T. Sugimoto, \& N.A. Nordin. (2015). Cellulose nanocrystals isolated from oil palm trunk Carbohydrate Polymers, 127, 202-208.

8. S. Bano, \& Y. S. Negi. (2017). Studies on cellulose nanocrystals isolated from groundnut shells. Carbohydrate Polymers, 157, 1041-1049.

9. D. Ye, \& J. Yang. (2015). Ion-responsive liquid crystals of cellulose nanowhiskers grafted with acrylamide. Carbohydrate Polymers, 134, 458-466.

10. F. Jiang, \& Y. L. Hsieh. (2013). Chemically and mechanically isolated nanocellulose and their self-assembled structures. Carbohydrate Polymers, 95, 32-40.

11. C. A. C. Mendes, N. M. S. Ferreira, C. R. G. Furtado, \& A. M. F. Sousa (2015). Isolation and characterization of nanocrystalline cellulose from cornhusk. Materials Letters, 148, 26-29.

\section{AUTHORS PROFILE}

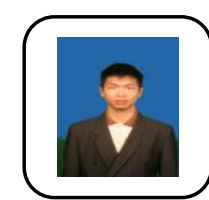

Lau Kia Kian is a postgraduate student working on research of nanocellulose particles. He has published a couple of papers related to nanocellulose fields. Currently, he is also writing some nanocellulose articles from other cellulose fibres.

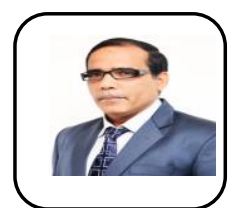

M. Jawaid is currently working as Fellow Researcher at Institute of Tropical Forestry and Forest Products (INTROP), Universiti Putra Malaysia. His area of expertise is polymer science. he has published 8 books, 25 book chapters, and more than 250 International journal papers. 